\title{
ASYMPTOTIC SOLUTION TO A CLASS OF SINGULARLY PERTURBED VOLTERRA INTEGRAL EQUATIONS
}

\section{A. Skinner}

\begin{abstract}
A uniformly valid asymptotic expansion for the solution to a class of singularly perturbed Volterra integral equations displaying exponential boundary layer behavior is established. Certain quasilinear ordinary differential equations are a noted special case, and a model for population growth with attrition is briefly discussed.
\end{abstract}

\section{Introduction}

Let $f(x, \epsilon) \in C^{\infty}([0,1] \times[0,1])$ and $k(x, t, \epsilon, y) \in C^{\infty}([0,1] \times[0, x] \times[0,1] \times[a, b])$, where $(a, b)$ contains the derivative $f_{\epsilon}(0,0)$, and assume $f(0,0)=0$. We are interested in the asymptotic behavior as $\epsilon \rightarrow 0^{+}$of the solution to the Volterra integral equation

$$
\epsilon y(x, \epsilon)+\int_{0}^{x} k(x, t, \epsilon, y(t, \epsilon)) d t=f(x, \epsilon) .
$$

The assumption $f(0,0)=0$, which implies $y(0, \epsilon)=f_{\epsilon}(0,0)+O(\epsilon)$, is nontrivial unless (1.1) is linear. If $k_{y y}(x, t, \epsilon, y)=0$, we can get $f(0,0)=0$ by changing the unknown (if necessary) to $\epsilon y(x, \epsilon)$.

In the special case

$$
\begin{gathered}
k(x, t, \epsilon, y)=p(t, \epsilon) y+(x-t)\left[q(t, \epsilon, y)-p_{t}(t, \epsilon)\right] y, \\
f(x, \epsilon)=\alpha \epsilon+[\beta \epsilon+\alpha p(0, \epsilon)] x,
\end{gathered}
$$

equation (1.1) is equivalent to the singularly perturbed initial-value problem

$$
\begin{gathered}
\epsilon y^{\prime \prime}+p(x, \epsilon) y^{\prime}+q(x, \epsilon, y)=0, \\
y(0)=\alpha, \quad y^{\prime}(0)=\beta .
\end{gathered}
$$

As is well known [5], if $p(x, 0)>0$ and the reduced problem

$$
p(x, 0) u^{\prime}+q(x, 0, u)=0, \quad y(0)=\alpha,
$$

has a solution for $0 \leq x \leq 1$, then the solution to (1.4)-(1.5) exists for $0 \leq x \leq 1$, and it has a uniformly valid asymptotic expansion of the form

$$
y(x, \epsilon)=\sum_{n=0}^{N-1} \epsilon^{n}\left[u_{n}(x)+v_{n}(x / \epsilon)\right]+O\left(\epsilon^{N}\right),
$$

where $u_{n}(x) \in C^{\infty}[0,1]$ and $v_{n}(X) \in C^{\infty}[0, \infty)$. Furthermore, $v_{n}(X)=o\left(X^{-\infty}\right)$. That is, $v_{n}(X)=o\left(X^{-r}\right)$ as $X \rightarrow \infty$ for any $r$. It can also be seen from the linear

Received June 29, 1994, revised December 5, 1994.

1991 Mathematics Subject Classification. 41A60, 45M05.

Key words and phrases: singular perturbation, Volterra integral equation, asymptotic expansion. 
Fredholm equation theory in [4] that the solution to (1.1) is expressible in the form (1.7) if $k(x, t, \epsilon, y)=c(x, t) y$ and $c(x, x)>0$.

The object of this paper is to establish the validity of (1.7), under appropriate conditions, for the full nonlinear problem (1.1). In so doing, we develop a procedure for successively computing the individual terms of (1.7). This procedure is rigorous and more explicit than the one presented in [1] and [2], which is a formal treatment of (1.1) and a variety of related problems including singular integral equations problems. We also briefly discuss a model for population growth with attrition.

Insisting that $v_{n}(X)=o\left(X^{-\infty}\right)$ in (1.7) may at first seem too restrictive. For instance, if

$$
k(x, t, \epsilon, y)=y^{2}-2 t y, \quad f(x, \epsilon)=\epsilon(1+x)-\frac{1}{3} x^{3},
$$

the solution to $(1.1)$ is $y(x, \epsilon)=x+(1+x / \epsilon)^{-1}$. However, this example is exceptional. Considering just the first term of (1.7), if we are to have $y(x, \epsilon)=u(x)+v(x / \epsilon)+O(\epsilon)$, then (1.1) implies

$$
u(0)+v(X)+\int_{0}^{X} k(0,0,0, u(0)+v(T)) d T=X f_{x}(0,0)+f_{\epsilon}(0,0) .
$$

Thus, in order to have $v(X)=O(1)$ for $0 \leq X<\infty$, it must be that $v(X)$ approaches an equilibrium point of

$$
v^{\prime}+k(0,0,0, u(0)+v)=f_{x}(0,0)
$$

as $X \rightarrow \infty$. Without loss of generality, $v(\infty)=0$, by choice of $u(0)$; therefore $k_{y}(0,0,0, u(0)) \geq 0$. If $k_{y}(0,0,0, u(0))>0$, then $k_{y}(0,0,0, y)>0$ in a neighborhood of $y=u(0)$; therefore $v(X) \rightarrow 0$ exponentially as $X \rightarrow \infty$. That is, $v(X)=o\left(X^{-\infty}\right)$ if $k_{y}(0,0,0, u(0))>0$. On the other hand, if $k_{y}(0,0,0, u(0))=0$, as in $(1.8)$, then $f_{x}(0,0)$ must just happen to be an extreme value of $k(0,0,0, y)$. Indeed, (1.10) also implies $k(0,0,0, u(0))=f_{x}(0,0)$. We shall therefore limit our investigation of (1.1) to solutions having asymptotic expansions of the form (1.7) with $v_{n}(X)=o\left(X^{-\infty}\right)$.

\section{Integral expansion}

Let

$$
s_{N}(t, T, \epsilon)=\sum_{n=0}^{N-1} \epsilon^{n}\left[u_{n}(t)+v_{n}(T)\right]
$$

where $u_{n}(t) \in C^{\infty}[0,1], v_{n}(T) \in C^{\infty}[0, \infty)$, and $v_{n}(T)=o\left(T^{-\infty}\right)$, but $u_{n}(t), v_{n}(T)$ are not yet tied to the solution of (1.1). We begin by determining a uniformly valid expansion for

$$
Y_{N}(x, \epsilon)=\int_{0}^{x} k\left(x, t, \epsilon, y_{N}(t, \epsilon)\right) d t
$$

where $y_{N}(t, \epsilon)=s_{N}(t, t / \epsilon, \epsilon)$. Note that we are presuming $a<u_{0}(t)+v_{0}(T)<b$ for all $(t, T) \in[0,1] \times[0, \infty)$, so that $y_{N}(t, \epsilon) \in(a, b)$ for $0 \leq t \leq 1$ and all sufficiently small $\epsilon>0$. We will use the following two theorems. 
Theorem 2.1. If $h(t, T, \epsilon) \in C^{\infty}([0,1] \times[0, \infty) \times[0,1])$ and $h(t, T, \epsilon)=o\left(T^{-\infty}\right)$ as $T \rightarrow \infty$, then

$$
h(t, t / \epsilon, \epsilon)=\sum_{n=0}^{N-1} \epsilon^{n} h_{n}(t / \epsilon)+O\left(\epsilon^{N}\right),
$$

where $h_{n}(T) \in C^{\infty}[0, \infty)$ is the coefficient of $\epsilon^{n}$ in the Taylor expansion of $h(\epsilon T, T, \epsilon)$, and $h_{n}(T)=o\left(T^{-\infty}\right)$.

Theorem 2.2. If $\phi(T, \epsilon) \in C^{\infty}([0, \infty) \times[0,1])$ and $g(t, \epsilon, y) \in C^{\infty}([0,1] \times[0,1] \times[a, b])$, where $a<\phi(T, \epsilon)<b$ for all $(T, \epsilon) \in[0, \infty) \times[0,1]$, and if $\phi(T, \epsilon)=o\left(T^{-\infty}\right)$, then

$$
g(t, \epsilon, \phi(t / \epsilon, \epsilon))=\sum_{n=0}^{N-1} \epsilon^{n}\left[g_{n}(t)+h_{n}(t / \epsilon)\right]+O\left(\epsilon^{N}\right),
$$

where $g_{n}(t) \in C^{\infty}[0,1]$ is the coefficient of $\epsilon^{n}$ in the Taylor expansion of $g(t, \epsilon, 0)$, $h_{n}(T) \in C^{\infty}[0, \infty)$ is the coefficient of $\epsilon^{n}$ in the expansion of $g(\epsilon T, \epsilon, \phi(T, \epsilon))$ $g(\epsilon T, \epsilon, 0)$, and $h_{n}(T)=o\left(T^{-\infty}\right)$.

Proofs. Let $p_{n}(t, T)$ be the coefficient of $\epsilon^{n}$ in the Taylor expansion of $h(t, T, \epsilon)$, and let $p_{n k}(T)$ be the coefficient of $t^{k}$ in the expansion of $p_{n}(t, T)$. Then $p_{n k}(T) \in C^{\infty}[0, \infty)$, $p_{n k}(T)=o\left(T^{-\infty}\right)$, and

$$
p_{n}(t, T)=\sum_{k=0}^{N-n-1} t^{k} p_{n k}(T)+t^{N-n} r_{N-n}(t, T),
$$

where $r_{N-n}(t, T) \in C^{\infty}([0,1] \times[0, \infty))$ and $r_{N-n}(t, T)=o\left(T^{-\infty}\right)$. Therefore $t^{N-n} r_{N-n}(t, t / \epsilon)=\epsilon^{N-n}\left[(t / \epsilon)^{N-n} r_{N-n}(t, t / \epsilon)\right]=O\left(\epsilon^{N-n}\right)$. Similarly, $t^{k} p_{n k}(t / \epsilon)=$ $\epsilon^{k} P_{n k}(t / \epsilon)$, where $P_{n k}(T)=T^{k} p_{n k}(T) \in C^{\infty}[0, \infty)$ and $P_{n k}(T)=o\left(T^{-\infty}\right)$. Thus, we have (2.3) with

$$
h_{n}(T)=\sum_{k=0}^{n} P_{k, n-k}(T) .
$$

Theorem 2.2 is obtained by applying Theorem 2.1 to

$$
h(t, T, \epsilon)=g(t, \epsilon, \phi(T, \epsilon))-g(t, \epsilon, 0) .
$$

By applying Theorem 2.2 to

$$
g(t, \epsilon, y, x)=k\left(x, t, \epsilon, \sum_{n=0}^{N-1} \epsilon^{n} u_{n}(t)+y\right)
$$

with

$$
\phi(T, \epsilon)=\sum_{n=0}^{N-1} \epsilon^{n} v_{n}(T)
$$

(and $x$ as an uninvolved parameter), it is apparent that

$$
k\left(x, t, \epsilon, y_{N}(t, \epsilon)\right)=\sum_{n=0}^{N-1} \epsilon^{n}\left[\phi_{n}(x, t)+\psi_{n}(x, t / \epsilon)\right]+O\left(\epsilon^{N}\right)
$$


where $\phi_{n}(x, t) \in C^{\infty}([0,1] \times[0, x]), \psi_{n}(x, T) \in C^{\infty}([0,1] \times[0, \infty))$, and $\psi_{n}(x, T)=$ $o\left(T^{-\infty}\right)$. A little computation also reveals

$$
\begin{aligned}
& \phi_{0}(x, t)=k\left(x, t, 0, u_{0}(t)\right) \\
& \phi_{1}(x, t)=k_{y}\left(x, t, 0, u_{0}(t)\right) u_{1}(t)+k_{\epsilon}\left(x, t, 0, u_{0}(t)\right)
\end{aligned}
$$

and, if we let

$$
h(x, t, \epsilon, y)=k\left(x, t, \epsilon, u_{0}(0)+y\right)-k\left(x, t, \epsilon, u_{0}(0)\right)
$$

then

$$
\begin{aligned}
& \psi_{0}(x, T)=h\left(x, 0,0, v_{0}(T)\right) \\
& \psi_{1}(x, T)=k_{y}\left(x, 0,0, u_{0}(0)+v_{0}(T)\right) v_{1}(T)+\widehat{\psi}_{1}(x, T)
\end{aligned}
$$

where $\widehat{\psi}_{1}(x, T)$ is the combination

$$
\widehat{\psi}_{1}(x, T)=\left(h_{\epsilon}+T h_{t}+u_{0}^{\prime}(0) T h_{y}+u_{1}(0) h_{y}\right)\left(x, 0,0, v_{0}(T)\right)
$$

In general, for $1 \leq n \leq N-1$,

$$
\begin{aligned}
& \phi_{n}(x, T)=k_{y}\left(x, t, 0, u_{0}(t)\right) u_{n}(t)+\widehat{\phi}_{n}(x, t) \\
& \psi_{n}(x, T)=k_{y}\left(x, 0,0, u_{0}(0)+v_{0}(T)\right) v_{n}(T)+\widehat{\psi}_{n}(x, T)
\end{aligned}
$$

where $\widehat{\phi}_{n}(x, t)$ and $\widehat{\psi}_{n}(x, T)-u_{n}(0) h_{y}\left(x, 0,0, v_{0}(T)\right)$ are determined by $u_{k}(t), v_{k}(T)$ for $0 \leq k \leq n-1$.

We need to substitute (2.10) into (2.2). Upon applying Theorem 2.1 to

$$
\Psi_{n}(x, X)=\int_{X}^{\infty} \psi_{n}(x, T) d T
$$

we get the desired result. Namely,

$$
Y_{N}(x, \epsilon)=\sum_{n=0}^{N-1} \epsilon^{n}\left[U_{n}(x)+\epsilon V_{n}(x / \epsilon)\right]+\epsilon^{N} \theta_{N}(x, \epsilon)
$$

where $U_{n}(x) \in C^{\infty}[0,1], V_{n}(X) \in C^{\infty}[0, \infty), V_{n}(X)=o\left(X^{-\infty}\right)$ and $\theta_{N}(x, \epsilon)=O(1)$ for $0 \leq x \leq 1$ as $\epsilon \rightarrow 0^{+}$. In particular,

$$
U_{0}(x)=\int_{0}^{x} k\left(x, t, 0, u_{0}(t)\right) d t, \quad V_{0}(X)=-\int_{X}^{\infty} h\left(0,0,0, v_{0}(T)\right) d T .
$$

For $1 \leq n \leq N-1$

$$
\begin{aligned}
U_{n}(x) & =\int_{0}^{x} k_{y}\left(x, t, 0, u_{n}(t)\right) d t+\widehat{U}_{n}(x) \\
V_{n}(X) & =-\int_{X}^{\infty} k_{y}\left(0,0,0, u_{0}(0)+v_{0}(T)\right) v_{n}(T) d T+\widehat{V}_{n}(X)
\end{aligned}
$$


where, letting $\lambda_{m n}(X)$ denote the coefficient of $x^{m}$ in the Taylor expansion of $\Psi_{n-m}(x, X)$,

$$
\begin{aligned}
\widehat{U}_{n}(x) & =\int_{0}^{x} \widehat{\phi}_{n}(x, t) d t+\Psi_{n-1}(x, 0) \\
\widehat{V}_{n}(X) & =-\int_{X}^{\infty} \widehat{\psi}_{n}(0, T) d T-\sum_{m=1}^{n} X^{m} \lambda_{m n}(X)
\end{aligned}
$$

In particular,

$$
\begin{aligned}
& \widehat{U}_{1}(x)=\int_{0}^{x} k_{\epsilon}\left(x, t, 0, u_{0}(t)\right) d t+\int_{0}^{\infty} h\left(x, 0,0, v_{0}(T)\right) d T \\
& \widehat{V}_{1}(X)=-\int_{X}^{\infty}\left[\widehat{\psi}_{1}(0, T)+X h_{x}\left(0,0,0, v_{0}(T)\right)\right] d T
\end{aligned}
$$

We could, of course, have absorbed $V_{N-1}(x / \epsilon)$ into $\theta_{N}(x, \epsilon)$ in (2.20). However, in its present form, since the expansion need not stop at $N$ terms, $\theta_{N}(x, \epsilon)=$ $p_{N}(x)+\epsilon \rho_{N}(x, \epsilon)$, where $p_{N}(x) \in C^{\infty}[0,1]$ and $\rho_{N}(x, \epsilon)=O(1)$ as $\epsilon \rightarrow 0^{+}$. Thus, we have the following key result.

Theorem 2.3. The derivative of the error term in $(2.20), \theta_{N x}(x, \epsilon)=O(1)$ for $0 \leq x \leq 1$ as $\epsilon \rightarrow 0^{+}$.

Proof. Just as we established (2.20) for $Y_{N}(x, \epsilon)$, we also have

$$
Y_{N x}(x, \epsilon)=\sum_{n=0}^{N} \epsilon^{n}\left[\widetilde{U}_{n}(x)+\widetilde{V}_{n}(x / \epsilon)\right]+\epsilon^{N+1} \widetilde{\theta}_{N+1}(x, \epsilon),
$$

where $\widetilde{U}_{n}(x) \in C^{\infty}[0,1], \widetilde{V}_{n}(X) \in C^{\infty}[0, \infty), \widetilde{V}_{n}(X)=o\left(X^{-\infty}\right)$, and $\tilde{\theta}_{N+1}(x, \epsilon)=$ $O(1)$ for $0 \leq x \leq 1$ as $\epsilon \rightarrow 0^{+}$, since

$$
Y_{N x}(x, \epsilon)=k\left(x, x, \epsilon, y_{N}(x, \epsilon)\right)+\int_{0}^{x} k_{x}\left(x, t, \epsilon, y_{N}(t, \epsilon)\right) d t
$$

Integrating (2.28) and comparing with (2.20) shows that

$$
\rho_{N}(x, \epsilon)=\int_{0}^{x / \epsilon} \tilde{V}_{N}(T) d T+\int_{0}^{x} \tilde{\theta}_{N+1}(t, \epsilon) d t .
$$

Therefore $\rho_{N x}(x, \epsilon)=\epsilon^{-1} \widetilde{V}_{N}(x / \epsilon)+\widetilde{\theta}_{N+1}(x, \epsilon)$, so $\theta_{N x}(x, \epsilon)=p_{N}^{\prime}(x)+\epsilon \rho_{N x}(x, \epsilon)=$ $O(1)$.

\section{Asymptotic solution}

In this section, we establish the preliminary result that, under appropriate conditions, $y_{N}(x, \epsilon)=s_{N}(x, x / \epsilon, \epsilon)$ satisfies (1.1) asymptotically, in the sense that

$$
\epsilon y_{N}(x, \epsilon)+Y_{N}(x, \epsilon)=f(x, \epsilon)-\epsilon^{N} \phi_{N}(x, \epsilon),
$$

where $\phi_{N}(x, \epsilon)=O(1)$ uniformly in $x$ for $0 \leq x \leq 1$ as $\epsilon \rightarrow 0^{+}$. From (2.1) and (2.20) it is clear that for (3.1) to hold, we must have

$$
u_{n-1}(x)+U_{n}(x)=f_{n}(x), \quad v_{n}(X)+V_{n}(X)=0,
$$


for $0 \leq n \leq N-1$, where $f_{n}(x)$ is the coefficient of $\epsilon^{n}$ in the Taylor expansion of $f(x, \epsilon)$ and $u_{-1}(x)=0$. Furthermore, (3.1) implies $u_{n}(0)+v_{n}(0)=f_{n+1}(0)$ and

$$
\phi_{N}(x, \epsilon)=\theta_{N}(x, \epsilon)+u_{N-1}(x)-\epsilon^{-N}\left[f(x, \epsilon)-\sum_{n=0}^{N-1} \epsilon^{n} f_{n}(x)\right],
$$

where $\theta_{N}(x, \epsilon)$ is the error term in (2.20). Thus, in light of Theorem $2.3, \phi_{N x}(x, \epsilon)=$ $O(1)$ as $\epsilon \rightarrow 0^{+}$. Also, $\theta_{N}(0, \epsilon)=-V_{N-1}(0)=v_{N-1}(0)$ by $(2.20)$ and (3.2), and thus $\phi_{N}(0, \epsilon)=v_{N-1}(0)+u_{N-1}(0)-\left[f_{N}(0)+0(\epsilon)\right]=O(\epsilon)$.

Of course, we need to show that (3.2) is consistent with the conditions imposed on $u_{n}(x)$ and $v_{n}(X)$ in deriving expansion (2.20). This requires some additional hypotheses, which we list below as $\mathrm{H} 2$ and $\mathrm{H} 3$. We have been assuming $\mathrm{H} 1$ all along.

H1. The function $f(x, \epsilon) \in C^{\infty}([0,1] \times[0,1])$, and $k(x, t, \epsilon, y) \in C^{\infty}([0,1] \times[0, x] \times$ $[0,1] \times[a, b])$. Also, $f_{0}(0)=0$ and $f_{1}(0) \in(a, b)$.

H2. The integral equation $U_{0}(x)=f_{0}(x)$ has a solution $u_{0}(x) \in C^{\infty}[0,1]$ with $a+\left|f_{1}(0)-u_{0}(0)\right|<u_{0}(x)<b-\left|f_{1}(0)-u_{0}(0)\right|$.

H3. There exists $\kappa>0$ such that $k_{y}\left(x, x, 0, u_{0}(x)\right) \geq \kappa$ for all $x \in[0,1]$, and $y=0$ is the only root of $h(0,0,0, y)$ in an interval containing $f_{1}(0)-u_{0}(0)$.

Condition H3 ensures $v_{0}(X)=o\left(X^{-\infty}\right)$. Indeed, $k_{y}\left(0,0,0, u_{0}(0)\right) \geq \kappa$ means $v_{0}=0$ is an attractor for $v_{0}^{\prime}+h\left(0,0,0, v_{0}\right)=0$, as noted in Section 1 , and the $h(0,0,0, y) \neq 0$ assumption means $v_{0}(0)=f_{1}(0)-u_{0}(0)$ is in the domain of attraction. Furthermore, H3 implies $v_{0}(X) \rightarrow 0$ monotonically, hence $a<u_{0}(x)+v_{0}(X)<b$.

For $1 \leq n \leq N-1$, the equations for $u_{n}(x)$ and $v_{n}(X)$ are linear. Indeed, (3.2), (2.22), and (2.23) imply

$$
\begin{gathered}
\int_{0}^{x} k_{y}\left(x, t, 0, u_{0}(t)\right) u_{n}(t) d t=f_{n}(x)-u_{n-1}(x)-\widehat{U}_{n}(x) \\
v_{n}^{\prime}+k_{y}\left(0,0,0, u_{0}(0)+v_{0}(X)\right) v_{n}=-\widehat{V}_{n}^{\prime}(X)
\end{gathered}
$$

for $1 \leq n \leq N-1$. Thus, by induction, $\widehat{U}_{n}(x) \in C^{\infty}[0,1]$, so, since $k_{y}\left(x, x, 0, u_{0}(x)\right)>$ $0, u_{n}(x) \in C^{\infty}[0,1]$. Also, $\widehat{V}_{n}(X) \in C^{\infty}[0, \infty)$ with $\widehat{V}_{n}(X)=o\left(X^{-\infty}\right)$, so $v_{n}(X) \in$ $C^{\infty}[0, \infty)$ and $v_{n}(X)=o\left(X^{-\infty}\right)$, since $k_{y}\left(0,0,0, u_{0}(0)+v_{0}(X)\right) \geq \kappa$ for $X$ sufficiently large.

Of course, regarding $\mathrm{H} 2, U_{0}(x)=f_{0}(x)$ may have no solution. For example, there is no solution if $k(x, t, 0, y)=y^{2}$ and $f(x, 0)=-x$. On the other hand, there can be at most one solution satisfying $k_{y}\left(x, x, 0, u_{0}(x)\right) \geq \kappa$. Indeed, there can be only one solution to (1.1) of the form (1.7).

If $k(x, t, \epsilon, y)=y^{3}-y$ and $f(x, \epsilon)=\epsilon(c+\epsilon)$, for example, then $U_{0}(x)=0$, since $f_{0}(x)=0$. Hence, referring to (2.21), there are three possibilities, namely, $u_{0}(x)= \pm 1$ and $u_{0}(x)=0$. However, if $u_{0}(x)=0$, then $v_{0}^{\prime}+v_{0}^{3}-v_{0}=0$ and $v_{0}(0)=c$. Thus, $v_{0}(X) \rightarrow \pm 1$ as $X \rightarrow \infty$, unless $c=0$. But if $c=0$, condition $\mathrm{H} 3$ fails to hold; indeed, the solution to (1.1) in this case, $y(x, \epsilon)=\epsilon /\left[\left(1-\epsilon^{2}\right) e^{-2 x / \epsilon}+\epsilon^{2}\right]$, is not expressible in the form (1.7). If $u_{0}(x)=1$, then $h\left(0,0,0, v_{0}\right)=v_{0}^{3}+3 v_{0}^{2}+2 v_{0}$ and $v_{0}(0)=c-1$, so $v_{0}(X)=o\left(X^{-\infty}\right)$ only if $c>0$. The remaining possibility, $u_{0}(x)=-1$, is the proper solution if $c<0$.

We conclude this section with a brief discussion of the population growth problem modeled by (1.1) when $f(x, \epsilon)=\epsilon s(x)$ and $k(x, t, \epsilon, y)=-s(x-t) y(1-y / c)$. In this 
model, the survival function $s(x)$ gives the fraction of the initial population which is still alive at time $x$ (so $\mathrm{s}(0)=1), y(x, \epsilon)$ is the (relative) total population size at time $x$, and $\epsilon^{-1} y(1-y / c)$ is the (rapid) rate of reproduction. Here we again have $U_{0}(x)=0$ and, thus, $u_{0}(x)=0$ or $u_{0}(x)=c$. To satisfy H3, we must take $u_{0}(x)=c$ and $c>0$. It follows that $v_{0}^{\prime}=-v_{0}\left(1+v_{0} / c\right), v_{0}(0)=1-c$. Therefore

$$
u_{0}(x)+v_{0}(X)=\frac{c}{1+(c-1) e^{-X}}
$$

which is notably independent of $s(x)$. In fact, $y_{0}(x, \epsilon)=u_{0}(x)+v_{0}(x / \epsilon)$ is the wellknown s-shaped exact solution to the differential equation form of this model which exists when $s(x)=1$.

With $y_{0}(x, \epsilon)$ determined, it follows from (3.4) and (2.26) that

$$
\int_{0}^{x} s(x-t) u_{1}(t) d t=-c[1-s(x)] .
$$

Hence $u_{1}(0)=c s^{\prime}(0)$; therefore, from (3.5) and (2.27),

$$
v_{1}^{\prime}+\left[1+(2 / c) v_{0}(x)\right] v_{1}=-s^{\prime}(0) v_{0}(x), \quad v_{1}(0)=-c s^{\prime}(0) .
$$

It is apparent from (3.7) that $u_{1}(x)<0$ for $x>0$. Thus, we see that the effect of attrition is that the population size never reaches the saturation level $y=c$. For instance, if $s(x)=(1+\alpha x) e^{-\alpha x}$, then

$$
u_{1}(x)=-(\alpha c / 2)\left(1-e^{-2 \alpha x}\right), \quad v_{1}(X)=0 .
$$

In addition to fertility being high, lifetimes are short in this example if $\alpha$ is large. Such problems are also studied in [3], but with the two rates connected. Obviously, here we would at least need $\alpha=o(1 / \epsilon)$.

To calculate $u_{2}(x)$ and $v_{2}(X)$, we would need to determine $\phi_{2}(x, t)$ and $\psi_{2}(x, T)$ in (2.10) and then use the fact that

$$
\begin{aligned}
U_{2}(x) & =\int_{0}^{x} \phi_{2}(x, t) d t+\int_{0}^{\infty} \psi_{1}(x, T) d T \\
V_{2}(X) & =-\int_{X}^{\infty}\left[\psi_{2}(0, T)+X \psi_{1 x}(0, T)+\frac{1}{2} X^{2} \psi_{0 x x}(0, T)\right] d T .
\end{aligned}
$$

\section{Confirmation}

We are in position now to prove that, under assumptions $\mathrm{H} 1-\mathrm{H} 3$, for all $\epsilon>0$ sufficiently small, (1.1) has a solution for $0 \leq x \leq 1$ with an asymptotic expansion given by (1.7), where $u_{n}(x)$ and $v_{n}(X)$ are the functions determined in Sections 2 and 3 . We shall follow a procedure patterned after the proof of comparable results for singularly perturbed ordinary differential equations. For example, see [6, pp. 197-205].

First, we consider the linear Volterra equation

$$
\epsilon z(x, \epsilon)+\int_{0}^{x} k_{y}\left(x, t, \epsilon, y_{N}(t, \epsilon)\right) z(t, \epsilon) d t=g(x, \epsilon) .
$$

We expect that if $y(x, \epsilon)$ is the solution to (1.1), then the difference $\epsilon^{-N}[y(x, \epsilon)-$ $y_{N}(x, \epsilon)$ ] nearly satisfies (4.1) when $g(x, \epsilon)=\phi_{N}(x, \epsilon)$, where $\phi_{N}(x, \epsilon)$ is the function in (3.1). 
Theorem 4.1. Choose $\epsilon_{0}>0$ so that $a<y_{N}(x, \epsilon)<b$ on $D=\{(x, \epsilon): 0 \leq x \leq 1$, $\left.0<\epsilon \leq \epsilon_{0}\right\}$. Assume $g(x, \epsilon)$ is continuously differentiable with respect to $x$ on $D$, and assume $g(0, \epsilon)=O(\epsilon), g_{x}(x, \epsilon)=O(1)$. Then the solution to (4.1) is uniformly bounded on $D$.

Proof. In terms of

$$
w(x, \epsilon)=g_{x}(x, \epsilon)-\int_{0}^{x} k_{x y}\left(x, t, \epsilon, y_{N}(t, \epsilon)\right) z(t, \epsilon) d t
$$

(4.1) is equivalent to

$$
\epsilon z^{\prime}+k_{y}\left(x, x, \epsilon, y_{N}(x, \epsilon)\right) z=w(x, \epsilon), \quad z(0, \epsilon)=\epsilon^{-1} g(0, \epsilon) .
$$

Thus, in terms of

$$
A(x, \epsilon)=\int_{0}^{x} k_{y}\left(s, s, \epsilon, y_{N}(s, \epsilon)\right) d s
$$

if we let

$$
\begin{gathered}
G(x, \epsilon)=\epsilon^{-1} g(0, \epsilon) e^{-A(x, \epsilon) / \epsilon}+\epsilon^{-1} \int_{0}^{x} e^{-[A(x, \epsilon)-A(t, \epsilon)] / \epsilon} g_{x}(t, \epsilon) d t \\
K(x, t, \epsilon)=\epsilon^{-1} \int_{t}^{x} e^{-[A(x, \epsilon)-A(t, \epsilon)] / \epsilon} k_{x y}\left(s, t, \epsilon, y_{N}(t, \epsilon)\right) d s
\end{gathered}
$$

it follows that (4.1) also is equivalent to

$$
z(x, \epsilon)+\int_{0}^{x} K(x, t, \epsilon) z(t, \epsilon) d t=G(x, \epsilon) .
$$

By an application of Theorem 2.2,

$$
k_{y}\left(s, s, \epsilon, y_{N}(s, \epsilon)\right)=k_{y}\left(s, s, 0, u_{0}(s)\right)+h_{y}\left(0,0,0, v_{0}(s / \epsilon)\right)+O(\epsilon) .
$$

Therefore

$$
A(x, \epsilon)-A(t, \epsilon)=\int_{t}^{x} k_{y}\left(s, s, 0, u_{0}(s)\right) d s+O(\epsilon)
$$

hence $A(x, \epsilon)-A(t, \epsilon) \geq \kappa(x-t)+O(\epsilon)$. Thus, we have

$$
\int_{0}^{x} e^{-[A(x, \epsilon)-A(t, \epsilon)] / \epsilon} g_{x}(t, \epsilon) d t=O(\epsilon)
$$

hence $G(x, \epsilon)=O(1)$ on $D$. Similarly, $K(x, t, \epsilon)=O(1)$ for $0 \leq x \leq 1,0 \leq t \leq x, 0<$ $\epsilon \leq \epsilon_{0}$. Hence, from (4.7), by an application of Gronwall's inequality, $z(x, \epsilon)=O(1)$ on $D$.

If we denote the solution to (4.1) by $z(x, \epsilon)=\mathcal{K} g(x, \epsilon)$, then $\mathcal{K}$ is a linear operator whose domain is all $g(x, \epsilon)$ satisfying the conditions of Theorem 4.1. Let $\mathbb{B}$ denote the Banach space of all $f(x) \in C^{0}[0,1]$ with $\|f\|=\max |f(x)|$, and let $S=\{f(x) \in \mathbb{B}:\|f\| \leq 2 d\}$, where $d$ is chosen so that $d>\left\|\mathcal{K} \phi_{N}(x, \epsilon)\right\|$ for $0<\epsilon \leq \epsilon_{0}$. For example, $f(x)=\epsilon x$ is in $S$ for any $\epsilon \leq 2 d$. Finally, let

$$
E(x, t, \epsilon, y, \rho)=\epsilon^{-2 N}\left[k\left(x, t, \epsilon, y+\epsilon^{N} \rho\right)-k(x, t, \epsilon, y)-\epsilon^{N} \rho k_{y}(x, t, \epsilon, y)\right],
$$


which is continuously differentiable. In particular, for $\epsilon_{0}$ sufficiently small, there exists $M \geq 0$ such that both

$$
\left|E\left(x, t, \epsilon, y_{N}(t, \epsilon), \rho\right)\right| \leq M \quad \text { and } \quad\left|E_{\rho}\left(x, t, \epsilon, y_{N}(t, \epsilon), \rho\right)\right| \leq M
$$

for all $(x, t, \epsilon, \rho) \in[0,1] \times[0, x] \times\left(0, \epsilon_{0}\right] \times[-2 d, 2 d]$.

Theorem 4.2. There exists $\epsilon_{0}>0$ such that the nonlinear problem

$$
\epsilon z(x, \epsilon)+\int_{0}^{x} F(x, t, \epsilon, z(t, \epsilon)) d t=\phi_{N}(x, \epsilon),
$$

where

$$
F(x, t, \epsilon, z)=k_{y}\left(x, t, \epsilon, y_{N}(t, \epsilon)\right) z+\epsilon^{N} E\left(x, t, \epsilon, y_{N}(t, \epsilon), z\right),
$$

has a solution in $S$, provided $0<\epsilon \leq \epsilon_{0}$.

Proof. Problem (4.13) is equivalent to $z(x, \epsilon)=\mathcal{N} z(x, \epsilon)$, where, for any $z(x, \epsilon) \in S$, the operator $\mathcal{N}$ is defined by

$$
\mathcal{N} z(x, \epsilon)=\mathcal{K}\left[\phi_{N}(x, \epsilon)-\epsilon^{N} \int_{0}^{x} E\left(x, t, \epsilon, y_{N}(t, \epsilon), z(t, \epsilon)\right) d t\right] .
$$

There exists $m>0$ such that $\|\mathcal{K} g(x, \epsilon)\| \leq m\|g(x, \epsilon)\|$ for any $g(x, \epsilon)$ in the domain of $\mathcal{K}$, hence $\|\mathcal{N} z(x, \epsilon)\| \leq d+\epsilon^{N} m M$ for any $z(x, \epsilon) \in S$. Thus, there exists $\epsilon_{0}>0$ such that, if $z(x, \epsilon) \in S$, then $\mathcal{N} z(x, \epsilon) \in S$ for $0<\epsilon \leq \epsilon_{0}$. Furthermore, since (4.12) also implies

$$
\begin{gathered}
\left|E\left(x, t, \epsilon, y_{N}(t, \epsilon), z(t, \epsilon)\right)-E\left(x, t, \epsilon, y_{N}(t, \epsilon), w(t, \epsilon)\right)\right| \\
\leq M|z(t, \epsilon)-w(t, \epsilon)|
\end{gathered}
$$

whenever $z(t, \epsilon), w(t, \epsilon) \in S$, we know

$$
\|\mathcal{N} z(x, \epsilon)-\mathcal{N} w(x, \epsilon)\| \leq m M \epsilon^{N}\|z(x, \epsilon)-w(x, \epsilon)\| .
$$

Thus, $\mathcal{N}$ is contracting on $S$ for $0<\epsilon \leq \epsilon_{0}<(m M)^{-1 / N}$.

Finally, we can state our main result, and its proof is now a simple computation.

Theorem 4.3. Let $R_{N}(x, \epsilon)$ be the solution to (4.13) determined by Theorem 4.2. Then $y(x, \epsilon)=y_{N}(x, \epsilon)+\epsilon^{N} R_{N}(x, \epsilon)$ satisfies (1.1) for $0 \leq x \leq 1,0<\epsilon \leq \epsilon_{0}$.

Proof. For $0 \leq x \leq 1,0 \leq t \leq x, 0<\epsilon \leq \epsilon_{0}$, we have

$$
k\left(x, t, \epsilon, y_{N}(t, \epsilon)+\epsilon^{N} R_{N}(t, \epsilon)\right)=k\left(x, t, \epsilon, y_{N}(t, \epsilon)\right)+\epsilon^{N} F\left(x, t, \epsilon, R_{N}(t, \epsilon)\right),
$$

and $y_{N}(x, \epsilon)$ satisfies (3.1). Therefore

$$
\begin{aligned}
& \int_{0}^{x} k\left(x, t, \epsilon, y_{N}(t, \epsilon)+\epsilon^{N} R_{N}(t, \epsilon)\right) d t \\
& \quad=f(x, \epsilon)-\epsilon^{N}\left[\phi_{N}(x, \epsilon)-\epsilon y_{N}(x, \epsilon)\right]+\epsilon^{N}\left[\phi_{N}(x, \epsilon)-\epsilon R_{N}(x, \epsilon)\right] .
\end{aligned}
$$




\section{References}

1. J. S. Angell and W. E. Olmstead, Singularly perturbed Volterra integral equations, SIAM J. Appl. Math. 47 (1987), 1-14.

2. __ Singularly perturbed Volterra integral equations II, SIAM J. Appl. Math. 47 (1987), 1150-1162.

3. F. C. Hoppensteadt, An algorithm for approximate solutions to weakly filtered synchronous control systems and nonlinear renewal processes, SIAM J. Appl. Math. 43 (1983), 834-843.

4. Charles G. Lange and Donald R. Smith, Singular perturbation analysis of integral equations, Stud. Appl. Math. 79 (1988), 1-63.

5. R. E. O'Malley, Jr., Singular Perburbation Methods for Ordinary Differential Equations, Springer-Verlag, New York, 1991.

6. Donald R. Smith, Singular Perturbation Theory, Camb. Univ. Press, Cambridge, 1985.

Department of Mathematical Sciences, University of Wisconsin, Milwaukee, Wi 53201, U.S.A. 